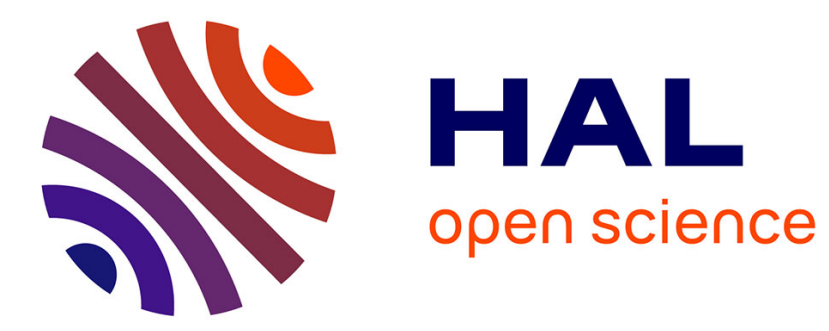

\title{
Liquid-solid 4He interface: Kapitza resistance
}

\author{
L. Puech, B. Hebral, D. Thoulouze, B. Castaing
}

\section{To cite this version:}

L. Puech, B. Hebral, D. Thoulouze, B. Castaing. Liquid-solid 4He interface: Kapitza resistance. Journal de Physique Lettres, 1982, 43 (22), pp.809-814. 10.1051/jphyslet:019820043022080900 . jpa00232129

\section{HAL Id: jpa-00232129 https://hal.science/jpa-00232129}

Submitted on 1 Jan 1982

HAL is a multi-disciplinary open access archive for the deposit and dissemination of scientific research documents, whether they are published or not. The documents may come from teaching and research institutions in France or abroad, or from public or private research centers.
L'archive ouverte pluridisciplinaire HAL, est destinée au dépôt et à la diffusion de documents scientifiques de niveau recherche, publiés ou non, émanant des établissements d'enseignement et de recherche français ou étrangers, des laboratoires publics ou privés. 
Classification

Physics Abstracts

67.80

\title{
Liquid-solid ${ }^{4} \mathrm{He}$ interface : Kapitza resistance
}

\author{
L. Puech, B. Hebral, D. Thoulouze and B. Castaing \\ Centre de Recherches sur les Très Basses Températures, \\ C.N.R.S., BP 166 X, 38042 Grenoble Cedex, France
}

(Reçu le 29 juin 1982, révisé le 20 septembre, accepté le 27 septembre 1982)

\begin{abstract}
Résumé. - Nous avons mesuré la résistance de Kapitza $R_{\mathrm{K}}$ entre les phases liquide et solide de ${ }^{4} \mathrm{He}$, de $100 \mathrm{mK}$ à $0,7 \mathrm{~K}$. Nous mettons en évidence la variation linéaire de $R_{\mathrm{K}} T^{3}$ avec $T^{-2}$ pour les interfaces rugueuses et l'anisotropie cristalline. L'influence de la tension de surface ne peut seule expliquer les résultats qui sont interprétés comme étant dus à l'inertie de l'interface. Pour les facettes on retrouve le résultat classique $R_{\mathrm{K}} T^{3}=$ Cte.
\end{abstract}

Abstract. - We have measured the Kapitza resistance $R_{\mathrm{K}}$ between liquid and solid ${ }^{4} \mathrm{He}$, from $100 \mathrm{mK}$ to $0.7 \mathrm{~K}$. We show that $R_{\mathrm{K}} T^{3}$ varies linearly with $T^{-2}$ for a rough interface and the crystalline anisotropy. The surface tension alone cannot explain the results which are interpreted as indicating an interface inertia. For the facets, the classical result is found : $R_{\mathrm{K}} T^{3}=$ constant.

1. Introduction - The Kapitza resistance $R_{\mathrm{K}}$ has been used for many years as a probe of the acoustic transmission across an interface. When this acoustic transmission is independent of the frequency of the phonons, it gives rise to the well known [1] temperature dependence

$$
R_{\mathrm{K}} T^{3}=\text { constant }
$$

In the case of the interface between liquid and solid ${ }^{4} \mathrm{He}$, this transmission is not usual. For most of the crystallographic orientations the solid can grow with a high interface mobility [2]. This reduces the acoustic transmission $[3,4]$ since the interface becomes nearly a node of pressure. Marchenko and Parshin [5] (M.P.) have suggested that, for an oblique incidence of the acoustic wave, the surface tension restores coupling at high frequency. The final result for the Kapitza resistance would be a stronger temperature dependence $R_{\mathrm{K}} \propto T^{-5}$ at low temperature for such rough interfaces. Alternatively, Puech and Castaing [6] have shown that such an anomalous behaviour can also be due to the " inertia " of the interface.

During the completion of this work, Huber and Maris reported measurements presenting a clear increase of $R_{\mathrm{K}} T^{3}$ when the temperature is lowered [7]. Depending on the sample their results seemed to indicate slightly increasing or decreasing $R_{\mathrm{K}} T^{5}$ with the temperature. They were however in fair agreement with a constant $R_{\mathrm{K}} T^{5}=2 \times 10^{-2} \mathrm{~cm}^{2} . \mathrm{K} / \mathrm{W}$, at low temperature, rather different from the value obtained from the M.P. theory : $R_{\mathrm{K}} T^{5}=0.1 \mathrm{~cm}^{2} . \mathrm{K}^{6} / \mathrm{W}[8]$.

By a different technique, we give further evidence for the dependence $R_{\mathrm{K}} \propto T^{-5}$. The quantitative disagreement with the M.P. result suggests that in fact the inertia of the interface plays the major role. With our better resolution, we have been able to study the effect of the crystallographic orientation. We have also shown that there is no anomalous dependence when the inter- 
face is a facet, which demonstrates that this effect originates in the high mobility of the interface. Finally, we have detected a transition of the interface that we suppose to be a faceting transition.

2. Experimental set up. - The cell has the same shape as the one used in previous experiments and already described [9]. The main part consists in two cylindrical $\mathrm{CuNi}$ tubes between which the crystal is grown at constant pressure (about 25 bars) (Fig. 1a). The dimensions are $8.5 \times 9 \mathrm{~mm}$ in diameter for the external tube and $5.5 \times 6 \mathrm{~mm}$ for the internal tube with length $7 \mathrm{~cm}$. The latter is filled by a graphited Vespel rod which holds 4 thermometers in direct contact with ${ }^{4} \mathrm{He}$. The distance between adjacent thermometers is $1 \mathrm{~cm}$.

The upper part of the cell is thermally connected to the mixing chamber of a dilution refrigerator.

The thermometers are Matsushita $68 \Omega, 1 / 8 \mathrm{~W}$ resistors. Their values, as well as their ratio which is necessary for this experiment [9], were measured using an a.c. bridge with optically decoupled signal injection [10]. Its sensitivity is better than $10 \mathrm{nV}$ which provides a resolution better than $10^{-4}$ with a current of $0.2 \mu \mathrm{A}$. During the measurements the overall $\Delta T$ in the cell was of the order of $1 \mathrm{mK}$. Heat leaks at the bottom of the cell have been measured to be of the order of $1 \mathrm{nW}$.

The resistances were calibrated using the fact that a fifth Matsushita $\left(R_{5}\right)$ of the same batch was in the mixing chamber, as well as a CMN thermometer. Comparing the variations of the resistances in the cell and $R_{5}$ versus the temperature, we detect the decoupling between the cell and the mixing chamber due to heat leaks. In this way, a difference of $0.3 \mathrm{mK}$ at $T=50 \mathrm{mK}$, between the cell and the mixing chamber, was clearly visible. Our uncertainty on the temperature is thus mainly due to the uncertainty on the $\mathrm{CMN}$ temperature, that is : $1 \%$.
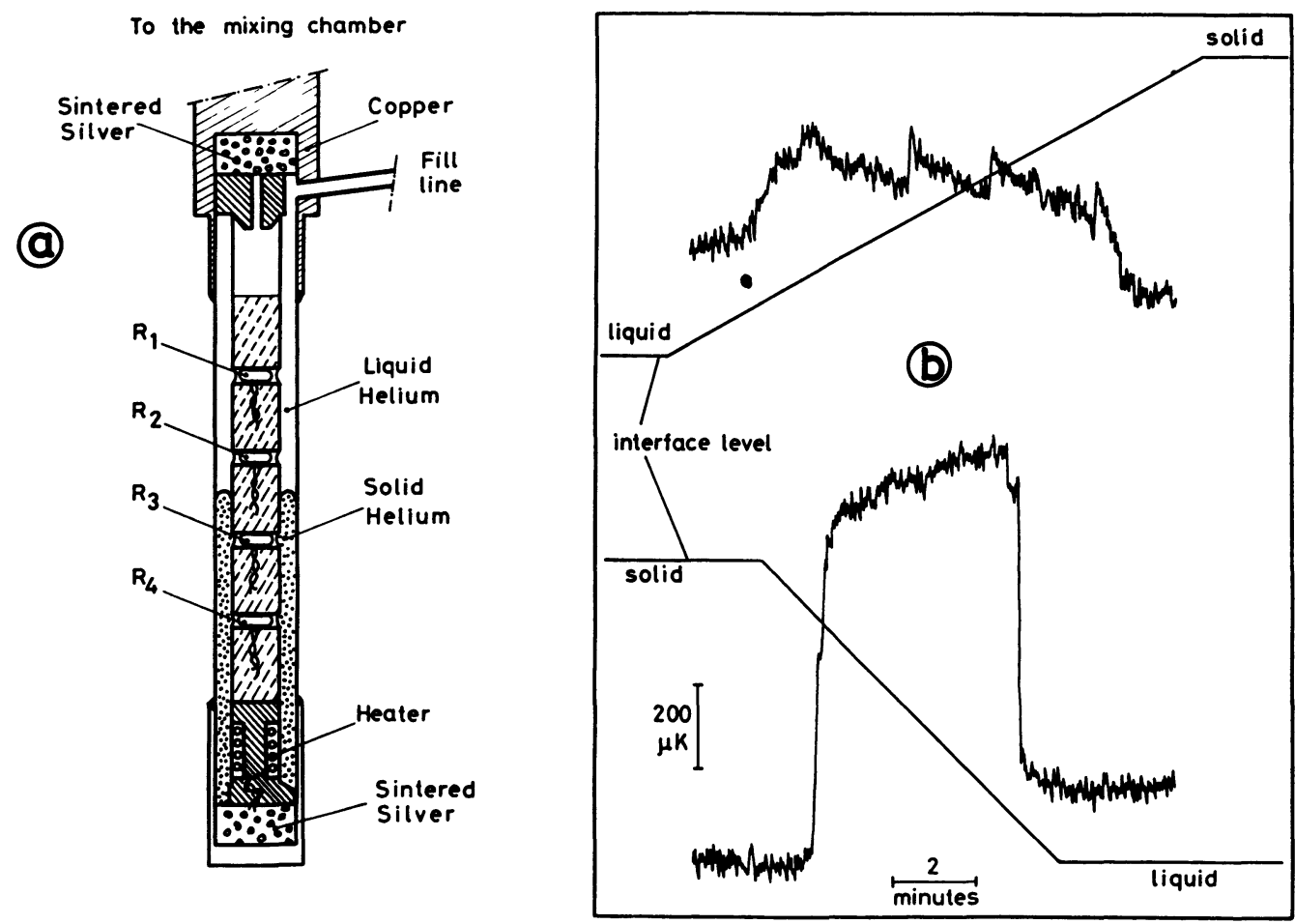

Fig. 1a. - The experimental cell.

Fig. 1b. - A typical experimental recording, $T=200 \mathrm{mK}$. Upper trace : when growing the crystal; Lower trace : when melting the crystal. 
3. Experimental procedure - In order to get rid of slow temperature drifts we used the fact that the ratio $R_{2} / R_{3}$ was nearly insensitive to the average temperature between $1 \mathrm{~K}$ and $50 \mathrm{mK}$ ( $1 \%$ of variation), while very sensitive to small temperature differences. This ratio is recorded during slow melting or crystallization (typically $1 \mathrm{~mm}$ per minute). A typical recording is shown in figure $1 b$.

On melting the crystal (lower trace), the temperature difference goes from its " all solid » value (giving the effective thermal conductivity of the solid) to its " all liquid " value. When the interface lies between $R_{2}$ and $R_{3}$ the additional Kapitza jump is clearly seen. The marked difference between growing (upper trace) and melting the crystal will be discussed in the next section.

In the high temperature Umklapp regime the anisotropy of the solid thermal conductivity allows us to determine the orientation of the crystals [11, 12]. We can also determine the density of dislocations in our solid which is of the order of $3 \times 10^{7}$ dislocations $/ \mathrm{cm}^{2}$ [13]. The complete study of the solid and liquid thermal conductivities will be given in a future paper [12].

4. Results. - At low temperature the equilibrium shape of the ${ }^{4} \mathrm{He}$ crystals shows facets (smooth interfaces) and rounded parts [14] (rough interfaces). When growing the size of the facets is enhanced due to their low mobility. The interface is no longer horizontal. In contrast during melting the facets disappear. The interface is well shaped, and its area is smaller. However the Kapitza jump is clearly higher (Fig. $1 b$ ). We shall first present the results on growing, then on melting.

4.1 FACETS. - On growing, when the facet is large enough to occupy nearly all the interface area, further growing can only occur by a jump of the facet which restores rounded parts. This corresponds to the jumps in $\Delta T$ clearly visible in figure $1 b$ (upper trace).

Evidently such a " relaxation » process can also occur before this extreme limit, but the smallest $\Delta T$ corresponds to an interface with a large facet, and is a measure of the Kapitza resistance of a facet.

The results are shown in figure $2 a$ (filled triangles). The product $R_{\mathrm{K}} T^{3}$ is constant within the experimental uncertainty as it must be for a classical interface. In this case no anomalous dependence of $R_{\mathrm{K}}$ is observed, which we interpret as being due to the fact that the mobility of a facet
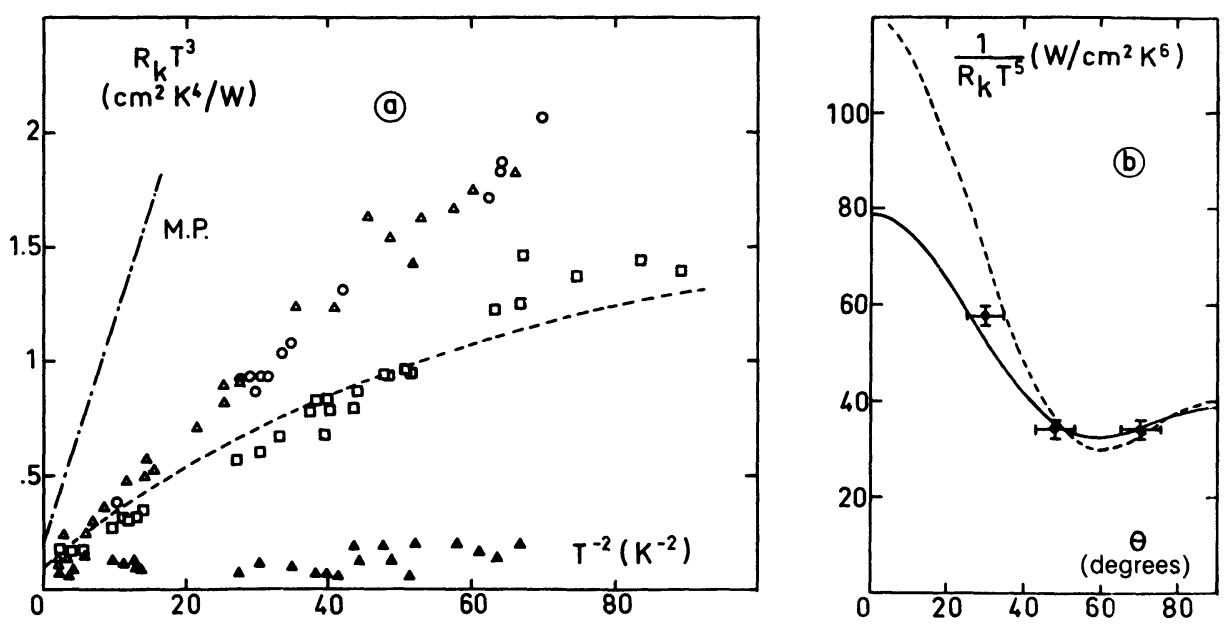

Fig. 2a. $-R_{\mathrm{K}} T^{3}$ as a function of $T^{-2}$ (linear scales). $\Delta$ Minimum values on growing the crystal. On melting the crystal $: \Delta: \theta \simeq 45^{\circ} ; O: \theta \simeq 80^{\circ} ; \square: \theta \simeq 30^{\circ}$.

Fig. 2b. - Example of anisotropy compatible with the experimental results. 
is small. We thus experimentally demonstrate that the anomalous dependence is related to the high mobility of the interface.

4.2 ROUGH INTERFACES. - In contrast, on melting, $R_{\mathrm{K}} T^{3}$ rises as $T$ decreases. It varies as $T^{-2}$. Such a $T^{-2}$ dependence was predicted by Marchenko and Parshin. However it is clear that the M.P. theory does not agree quantitatively with the results. We have made several runs with crystals whose orientation was randomly obtained, and determined by the thermal conductivity anisotropy. In this determination, half of the error came from the measurement of the reference (zero heat flux) value of the ratio $R_{2} / R_{3}$. Thus the relative angles are better determined than are their absolute value.

We must emphasize that, for these results, no correction due to the meniscus shape has been taken into account. According to Keshishev et al. [14] the contact angle lies between $60^{\circ}$ and $30^{\circ}$. The supplementary area would give $R_{\mathrm{K}}$ higher by $5 \%$ to $15 \%$.

We thus had access to the angle $\theta$ between the $c$-axis and the axis of the cell. The results noted $\triangle$ correspond to : $\theta=45^{\circ} \pm 10^{\circ}$ and the result noted $\square$, to : $\theta=30^{\circ} \pm 10^{\circ}$. The open circles correspond to several runs. The orientation of one of them was measured to be : $\theta=80^{\circ} \pm 10^{\circ}$.

For the highest results $(\triangle, O)$ the mean slope is :

$$
R_{\mathrm{K}} T^{5}=(2.85 \pm 0.3) \cdot 10^{-2} \mathrm{~cm}^{2} \cdot \mathrm{K}^{6} / \mathrm{W}
$$

(the error corresponds to the absolute determination).

Interpreted as due to the inertia of the interface [6] it corresponds to :

$$
\sigma=1.95 \times 10^{-9} \mathrm{~kg} / \mathrm{m}^{2} \text {. }
$$

The order of magnitude will appear even more clearly if we put it in the form :

$$
\frac{\sigma}{\rho_{\mathrm{S}}}=0.102 \AA \text {. }
$$

Two interpretations are possible for the lowest results $(\square)$ : the first is to assume that $R_{\mathrm{K}}$ (and thus $\sigma$ ) is in fact isotropic, but that, due to the proximity of the $c$-axis, a facet was maintained, even when melting [15]. The dotted line corresponds to such an interpretation where the facet takes $4 \%$ of the interface area.

The results differ significantly from this fit. Moreover the area of the facet would depend on the velocity of the interface. The results would thus be randomly distributed between the two extreme slopes. Our interpretation is that the difference in slope corresponds to the anisotropy of $\sigma$. In this case $\left(\theta \simeq 30^{\circ}\right)$ :

$$
R_{\mathrm{K}} T^{5}=(1.68 \pm 0.15) \cdot 10^{-2} \mathrm{~cm}^{2} \cdot \mathrm{K}^{6} / \mathrm{W}
$$

which corresponds to : $\sigma=2.53 \times 10^{-9} \mathrm{~kg} / \mathrm{m}^{2}$, or $\frac{\sigma}{\rho_{\mathrm{s}}}=0.133 \AA$.

As shown in figure $2 b$, it is reasonably possible to observe such an anisotropy, even with the averaging effect of the meniscus shape. We have plotted $1 /\left(R_{\mathrm{K}} T^{5}\right)$ versus $\theta$. Clearly the observations are compatible with the full line. Assuming that the meniscus is part of a torus, with a contact angle of $45^{\circ}$ [14], this full line corresponds to the average of the anisotropy represented by the dotted line. Such an anisotropy would give a factor 2 between the lowest and the highest values of $\sigma$. This is not surprising, Keshishev $e t$ al. [14] have reported such a factor 2 for the surface tension $\tilde{\alpha}$.

4.3 FACETING TRANSITION. - For the last sample (Fig. 3), the orientation is $\theta=75^{\circ} \pm 10^{\circ}$. On melting the results coincide with the highest values in figure $2 a$. 


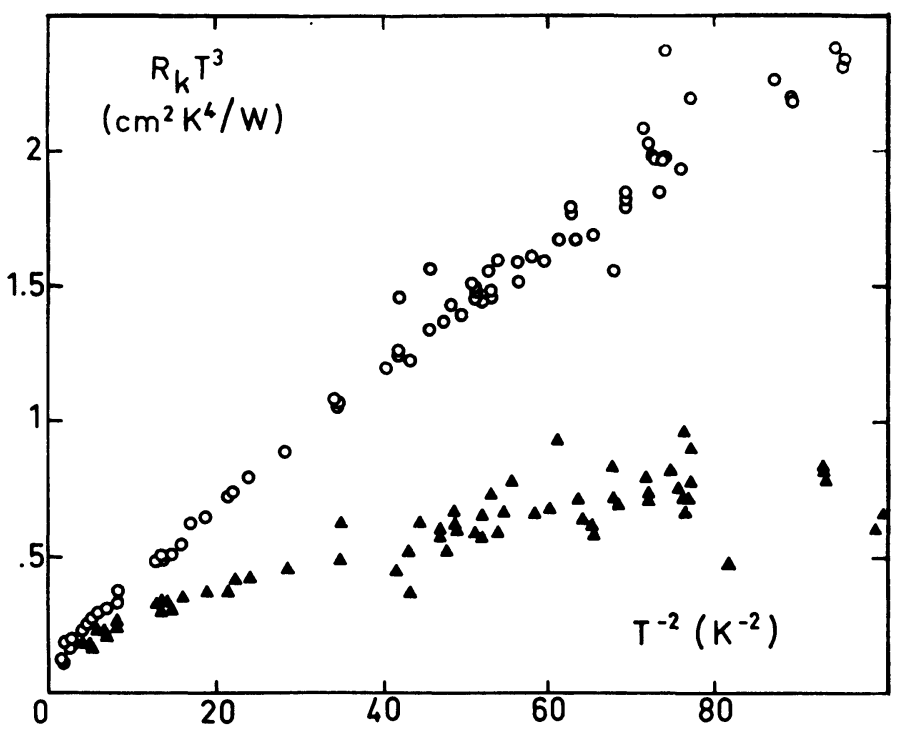

Fig. 3. - The special case showing a faceting transition. $\theta \simeq 75^{\circ}: \Delta$ on growing; $\bigcirc$ on melting.

In contrast, on growing, the results are clearly different. This can be due to the fact that the facets are small, even on growing, or that their mobility is so high that their own Kapitza resistance is enhanced.

In fact both explanations imply a high mobility for the facet, that is, a high density of steps on it. Close to $\theta=70^{\circ}$, P. E. Wolf and S. Balibar [15] have observed a new facet on ${ }^{4} \mathrm{He}$ crystals, in the neighbourhood of $T=200 \mathrm{mK}$. The existence of this faceting transition would justify the high density of steps.

We have confirmed the presence of this transition by observing a critical slowing down of the relaxation of the interface towards its equilibrium shape. A detailed analysis of this phenomenon will be given in a future publication.

5. Conclusion - These new results on the Kapitza resistance between liquid and solid ${ }^{4} \mathrm{He}$ agree with the dynamic results of Huber and Maris to within their experimental uncertainty. The precision of our method allows us to establish the $T^{-2}$ linear dependence of $R_{\mathrm{K}} T^{3}$ and its crystallographic anisotropy. It allows us, moreover, to demonstrate that for a facet the Kapitza resistance has the classical behaviour : $R_{\mathrm{K}} T^{3}=$ constant. This appears to provide a good way of studying the faceting transition close to $200 \mathrm{mK}$.

Finally, our results also clearly establish that the transmission of high frequency phonons is not governed by the surface tension, but probably by the inertia of the interface. A complete proof could be given, as already suggested [8], by the study of the transmission versus the incident angle for nearly monochromatic phonon beams.

Acknowledgments. - We are grateful to T. Huber and H. Maris for communicating their results prior to publication. We thank R. Fagaly for his contribution during the rebuilding of the dilution unit. 


\section{References}

[1] Khalatnikov, I. M., An Introduction to the theory of superfluidity (Benjamin Press, New York, N. Y.) 1965.

[2] Andreev, A. F. and Parshin, A. Ya., Zh. Eksp. Teor. Fiz. 75 (1978) 151 (Sov. Phys. J.E.T.P. 48 (1978) 763).

[3] Castaing, B. and Nozieres, P., J. Physique 41 (1980) 701.

[4] Castaing, B., Balibar, S. and Laroche, C., J. Physique 41 (1980) 897.

[5] Marchenko, V. I. and Parshin, A. Ya., Pis'ma Zh. Eksp. Teor. Fiz. 31 (1980) 767 (J.E.T.P. Lett. 31 (1980) 724).

[6] Puech, L. and Castaing, B., J. Physique-Lett. 43 (1982) L-601.

[7] Huber, T. E. and MARIS, H. J., Phys. Rev. Lett. 47 (1981) 1907.

[8] Huber, T. E. and Maris, H. J., J. Low Temp. Phys. 48 (1982) 99.

[9] Castaing, B., Bonfait, G. and Thoulouze, D., Proceedings of L.T. 16 (Physica, North Holland Publishing Company) 1982.

[10] Bret, J. L. and Faure, J. P., this bridge was a modified version of the 4-wires bridge model P.I.O. developed in CRTBT-CNRS and commercialized by Barras Provence, 12 rue Ampère, 38000 Grenoble, France.

[11] Hogan, E. M., Guyer, R. A. and Fairbanks, H. A., Phys. Rev. 185 (1969) 356.

[12] Puech, L., Hebral, B., Thoulouze, D. and Castaing, B., to be published.

[13] Golub, A. A. and Svatko, S. V., Fiz. Nizk. Temp. 6 (1980) 957 (Sov. J. Low Temp. Phys. 6 (1980) 465).

[14] Landau, J., Lipson, S. G., Mä̈̈ttänen, L. M., Balfour, L. S. and Edwards, D. O., Phys. Rev. Lett. 45 (1980) 31.

Keshishev, K. O., Parshin, A. Ya. and Babkin, A. B., Zh. Eksp. Teor. Fiz. 80 (1980) 716 (Sov. Phys. J.E.T.P. 53 (1981) 362).

[15] Balibar, S., Private communication. 